\title{
Bildgebung 2005 - Thoraxsonographie
}

\author{
Imaging 2005 - Ultrasound of the Chest
}

Während die sonographische Untersuchung von Pleuraergüssen bereits seit den 60er-Jahren als Routinemethode etabliert ist, erobert die Sonographie der Thoraxorgane gegenwärtig eine Vielzahl diagnostischer Zugänge. Diese sind im Einzelnen: die Echokardiographie, die Endosonographie via Ösophagus/Magen (EUS), die endobronchiale Sonographie (EBUS), die intraoperative bzw. die thorakoskopische Sonographie und die transthorakale Sonographie der Lunge und Pleura (TS) einschließlich Mediastinalsonographie. Ziel dieser Übersicht ist es, die diagnostischen Möglichkeiten der Sonographie im Bereich des Thorax zusammenzufassen.

\section{Sonographische Methoden}

Die Echokardiographie liefert auch für Pneumologen klinisch relevante Informationen, insbesondere wenn es um den Ausschluss kardialer Erkrankungen oder die Diagnostik einer akuten oder chronischen Rechtsherzbelastung geht. So kommt der Technik, z.B. bei der Beurteilung der Schwere einer Lungenembolie, eine wichtige Rolle zu. Neben der gelegentlichen Darstellung zentraler Thromben lässt sich echokardiographisch eine Untergruppe von Patienten mit submassiver Lungenembolie identifizieren. Patienten mit submassiver Lungenembolie weisen eine Manifestationsform der nicht-massiven Lungenembolie ohne Schocksymptomatik auf, bei der sich echokardiographisch eine rechtsventrikuläre Hypokinesie findet. Wie verschiedene Studien zeigen, haben diese Patienten ein erhöhtes Mortalitätsrisiko und bedürfen daher einer intensivmedizinischen Überwachung und in Abhängigkeit vom Verlauf, von kardialen Biomarkern sowie unter Beachtung der Kontraindikationen gegebenenfalls einer systemischen Thrombolyse [1,2].
Die Endosonographie (via Ösophagus/Magen) mit Punktion (EUS-FNA) ist heute nicht mehr aus der Diagnostik und dem Staging von Bronchialkarzinomen wegzudenken. Mit Hilfe der EUSFNA können die mediastinalen Lymphknoten ebenso wie die linke Nebenniere nicht nur beurteilt, sondern auch sonographischgeführt punktiert und somit zytologisch bzw. histologisch abgeklärt werden. Während computertomographisch Lymphknoten mit einer Größe über $1 \mathrm{~cm}$ als tumorsuspekt gelten, ist es endosonographisch möglich, auch kleinere Lymphknoten nachzuweisen und im gleichen Untersuchungsgang feingeweblich abzuklären. Gelegentlich erlaubt die EUS auch die Darstellung und Diagnosesicherung des Primärtumors.

Zur zytologisch-histologischen Abklärung des mediastinalen Lymphknotenstatus lassen sich verschiedene, zueinander komplementäre Techniken einsetzen. Neben der EUS kommt die konventionelle transbronchiale Nadelaspiration (TBNA) (ohne Ultraschalllokalisation) ebenso wie die endobronchiale Sonographie und die Mediastinoskopie infrage. Seit kurzem steht ein Ultraschall-Punktionsbronchoskop für die endobronchiale Sonographie zur Verfügung, mit dem sonographisch auffällige Prozesse auch endobronchial direkt unter Sicht punktiert werden können. Durch den Einsatz der EUS und der endobronchialen Sonographie mit Nadelaspiration lassen sich die Kosten für das Staging ebenso wie die Invasivität der Untersuchung und der erforderliche Zeitaufwand deutlich vermindern. Haupteinsatzgebiet der endobronchialen Sonographie mittels Minisonden ist dagegen die diagnostische Abklärung peripherer Lungenrundherde.

Die intraoperative Sonographie oder thorakoskopische Sonographie komplettiert die sonographischen Zugangswege im Bereich der Lunge und des Thorax. Die videoassistierte Thorakoskopie

Serienherausgeber: C. Witt, U. Costabel

Institutsangaben

Pneumologie und Allergologie, Klinik für Innere Medizin I, Friedrich-Schiller-Universität

Korrespondenzadresse

OÄ Dr. med. Angelika Reißig · Pneumologie \& Allergologie · Klinik für Innere Medizin I · Friedrich-SchillerUniversität ·Erlanger Allee $101 \cdot 07740$ Jena ·E-mail: angelika.reissig@med.uni-jena.de

Bibliografie

Pneumologie 2005; 59: 477-484 @ Georg Thieme Verlag KG Stuttgart · New York DOI $10.1055 / \mathrm{s}-2005-870946$ 
(VATS) erlaubt keine palpative Exploration der Lunge, wodurch die Identifikation insbesondere kleiner Rundherde erschwert wird. Hier stellt die thorakoskopische Sonographie eine Erweiterung der diagnostischen Möglichkeiten dar. Intraoperativ wird der Ultraschall gelegentlich zur Festlegung des Umfangs einer Operation eingesetzt. Das gilt vor allem für die Beurteilung der Ausdehnung eines Malignoms mit Invasion von Gefäßen oder anderen Thoraxstrukturen.

\section{Transthorakale Sonographie der Lunge und Pleura}

Die transthorakale Sonographie der Lunge und Pleura einschließlich Mediastinalsonographie ist in den letzten Jahren zu einem festen Bestandteil der modernen pneumologischen Diagnostik geworden [3-6]. Mit ihrer Hilfe können alle pulmonalen Veränderungen, die bis zur Pleura visceralis heranreichen oder die über ein so genanntes Schallfenster (z. B. Erguss oder Atelektase) erfassbar sind, dargestellt werden.

\section{Apparative Voraussetzungen}

Für die transthorakale sonographische Untersuchung der Pleura und des Lungenparenchyms eignen sich die heute üblichen Ultraschallgeräte mit hochauflösender „Echtzeit-B-Mode“-Technik. Bei bestimmten Fragestellungen ist der Einsatz der farbkodierten Duplexsonographie bzw. des „Power Mode“ sinnvoll.

Als Schallsonden kommen sowohl lineare, konvexe als auch Sektorscanner in Betracht. Dabei hängt die Auswahl der verwendeten Schallsonden von der Lokalisation des zu untersuchenden Prozesses ab. Mit Hilfe des niedrigfrequenten Konvexscanners (3,5 MHz) lässt sich zunächst ein Überblick verschaffen. Der höherfrequente Linearscanner ( $7,5 \mathrm{bis} 10 \mathrm{MHz}$ ) erlaubt eine detaillierte Darstellung im Nahbereich, d.h. im Bereich der Thoraxwand sowie der Pleura- und Lungenoberfläche. Eine an die jeweilige Fragestellung angepasste Geräteeinstellung ist erforderlich.

\section{Limitationen}

Der Einsatz der transthorakalen Sonographie wird durch bestimmte methodische und anatomische Gegebenheiten limitiert, deren sich der Untersucher bewusst sein sollte. Das sind in erster Linie

- die Totalreflexion der Schallwellen am Übergang zu lufthaltigem Gewebe sowie

- die Absorption der Schallwellen, z. B. durch knöcherne Strukturen.

Aus diesem Grunde entziehen sich sowohl innerhalb des Lungengewebes als auch subskapulär, paravertebral, retrosternal und im hinteren Mediastinum gelegene Prozesse einer sonographischen Darstellung. Damit sind etwa $60-70 \%$ der Pleuraoberfläche der transthorakalen Beurteilung zugänglich. Darüber hinaus erlaubt die transthorakale Sonographie im Gegensatz zu den radiologischen Verfahren nur die Darstellung eines definierten Ausschnittes, so dass eine integrierende Beurteilung in Beziehung zu anderen Thoraxorganen nur eingeschränkt möglich ist. Ferner hängt die Interpretation und Reproduzierbarkeit der sonographischen Befunde von den Erfahrungen des jeweiligen

Untersuchers, Art und Umfang der Dokumentation sowie von der Vollständigkeit der Untersuchung ab.

\section{Indikationen und Möglichkeiten}

Aufgrund der fehlenden Invasivität kann die Indikation zur Lungen- und Pleurasonographie großzügig gestellt werden. Die Untersuchung ist bei immobilen Patienten auch direkt am Krankenbett möglich. Dieses Vorgehen wird durch die Entwicklung moderner, tragbarer Ultraschallgeräte in den letzten Jahren noch weiter begünstigt. Andere Vorteile der Sonographie sind die Verbreitung bzw. generelle Verfügbarkeit der Technik, die „Real-Time“-Untersuchung, die Beurteilung der Vaskularisation bei Einsatz des Farbdopplers und die Möglichkeit der beliebigen Wiederholung einschließlich der Anwendung während der Schwangerschaft und bei Kontrastmittelallergie. Darüber hinaus lässt sich eine transthorakale Punktion oder Biopsie mit einer Untersuchung unmittelbar kombinieren. Schließlich können sonographisch erfassbare Prozesse beliebig oft mittels der Methode nachkontrolliert werden. Die wichtigsten Indikationen für die transthorakale Sonographie sind in Tab.1 zusammengefasst.

\section{Untersuchungsablauf}

Die transthorakale Sonographie der Lunge und Pleura erfolgt in der Regel von dorsal am sitzenden und von ventral am liegenden Patienten. Bei immobilen Patienten kann die Sonographie aus-

Tab. 1 Indikationen zur transthorakalen Sonographie der Lunge und Pleura (modifiziert nach [3])

Indikation*

atemabhängige Thoraxschmerzen

Weichteilveränderungen unklarer Ätiologie

Dyspnoe unklarer Genese

\section{Thoraxtrauma}

Fieber unklarer Genese Klopfschalldämpfung

abgeschwächtes Atemgeräusch

Zwechfellhochstand/

Phrenicusparese

periphere Lymphadeno-

pathie

Verdacht auf Pleuritis

Verdacht auf Lungenembolie

Verdacht auf Pneumothorax

Verdacht auf peripheres

Bronchialkarzinom

Verdacht auf Pleuratumor/

Pleuramesotheliom

Verdacht auf Pneumonie

Verdacht auf Knochenfraktur bzw. Osteolysen

\footnotetext{
* Bei allen Indikationen ist die Verlaufskontrolle mittels Sonographie möglich.
}

Lungenembolie, Pleuritis, Pleuropneumonie, Tumorinfiltration, Frakturen, Osteolysen benigne und maligne Tumoren, Lymphadenopathie, Hämatom

Pleuraerguss, Pleuraempyem, Pneumonie, Lungenembolie, Bronchialkarzinom, Atelektase, Pleuramesotheliom, Pleuraschwarte, interstitielle Lungenerkrankungen, Perikarderguss

Pneumothorax, Lungenkontusion, Pleuraerguss/Hämatothorax, Rippenfraktur Pneumonie, Lungenabszess, Pleuraempyem

Pleuraerguss, Pleuraschwarte, Bronchialkarzinom

Pleuraerguss, Pleuraschwarte, Bronchialkarzinom

Diaphragma-Lage und Mobilität

Ausdehnung, Hinweise auf benigne oder maligne Genese

diagnostische Bestätigung

diagnostische Bestätigung

diagnostische Bestätigung

diagnostische Bestätigung, gegebenenfalls

Punktion

diagnostische Bestätigung

diagnostische Bestätigung

diagnostische Bestätigung 
schließlich am liegenden Patienten durchgeführt werden. Der Patient wird dann zur Beurteilung der dorsalen Lungen- und Pleuraanteile in eine Seitlagerung gebracht. In Abhängigkeit von der Fragestellung besteht die Möglichkeit einer gezielten lokalen Untersuchung (z. B. bei der Frage nach Vorliegen eines Pleuraergusses) oder einer Beurteilung aller ventralen und dorsalen Interkostalräume (z. B. bei Verdacht auf Lungenembolie). Durch Anheben der Arme und Verschränken hinter dem Kopf lassen sich die Interkostalräume erweitern. Der subscapuläre Bereich wird durch die Außenrotation der Scapula partiell darstellbar.

\section{Transthorakale Sonographie ausgewählter pneumologischer Krankheitsbilder}

Die transthorakale Sonographie der Lunge und Pleura lässt sich bei einer Vielzahl pneumologischer Erkrankungen einsetzen (Tab.1). Die wichtigsten sollen nachfolgend dargestellt werden.

\section{Bronchialkarzinom}

Bei der Karzinogenese wird normales lufthaltiges Gewebe durch solides Tumorgewebe ersetzt bzw. verdrängt. Durch den fehlenden Luftgehalt wird das Tumorgewebe bei subpleuraler Lage der sonographischen Untersuchung zugänglich. Tumoren lassen sich als echoarme oder mäßig echodichte, polyzyklische (Abb.1), runde oder auch ovale Prozesse sonographisch darstellen. Die Echotextur ist variabel und reicht von einer homogenen bis zu einer inhomogenen Binnenstruktur. Die Randbereiche sind meist unregelmäßig strukturiert und lassen gelegentlich echoarme pseudopodienartige Tumorausläufer erkennen, die entweder auf eine regionale Lymphangiosis oder auf ein lokales Wachstum zurückgehen. Ein Vorteil der Sonographie ist die „Real-Time“-Beurteilung der Atemverschieblichkeit von Prozessen. Dadurch können Tumoren, die die Thoraxwand einbeziehen und dann nicht mehr atemverschieblich sind, mit hoher Sensitivität erfasst werden. Hinsichtlich der Beurteilung einer malignen Infiltration der Pleura bzw. Thoraxwand ist die Sonographie der konventionellen Röntgenaufnahme überlegen. Sie besitzt diesbezüglich eine mit der Computertomographie vergleichbare Sensitivität [7].

Auch Tumoreinschmelzungen lassen sich sonographisch erkennen. Sie imponieren in der Regel als echoarmes bis echofreies Areal innerhalb des echoarmen malignen Prozesses. In einer Nekrosehöhle finden sich gelegentlich echogene Reflexe, die Ausdruck „gefangener“ Luft sind. Weniger echoarme, unregelmäßige Strukturen bilden das Korrelat für Gewebesequester. Echoreiche Pleuraergüsse weisen auf eine maligne Genese hin und können, sonographisch gestützt, sofort punktiert werden. Pleurametastasen stellen sich sonographisch als echoarme, Pleura-ständige Strukturen dar, die sich im Pleuraerguss gut differenzieren und auch punktieren lassen.

Sonographisch darstellbare Tumoren sollten unter sonographischer Kontrolle punktiert bzw. bioptiert werden. Die sonographisch geführte transthorakale Biopsie ist für die Diagnostik peripherer Lungenherde von außerordentlichem Wert und ergänzt die Möglichkeiten des bronchoskopischen Zugangs. Gegenüber der computertomographisch gesteuerten Punktion besteht ihr Vorteil vor allem in der „Real-time“-Beobachtung des Punktionsvorganges und in der dreidimensionalen Darstellbarkeit der Prozesse [8]. Außerdem kann unmittelbar nach erfolgter Biopsie

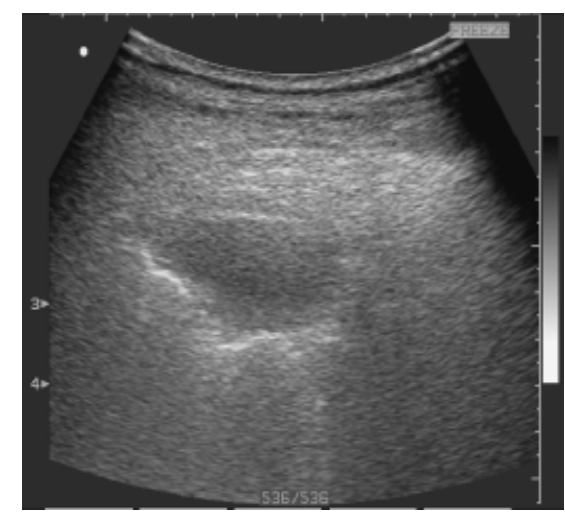

Abb. 1 Adenokarzinom - Sonogramm (Konvexscanner). Es zeigt sich ein polygonaler, echoarmer, homogen strukturierter Prozess, der in der „Real-Time“-Untersuchung keine Atemverschieblichkeit aufwies.

eine Aussage hinsichtlich evt. aufgetretener Komplikationen (z.B. Blutung oder Pneumothorax) getroffen werden. Die Treffsicherheit sonographisch geführter Punktionen liegt bei Karzinomen und Metastasen zwischen 70 und 97\% (durchschnittlich deutlich über $90 \%$ ), für benigne Veränderungen liegt die Trefferrate bei 70\% [9]. Die Indikation für eine CT-gestützte Punktion ist auf die Fälle begrenzt, bei denen eine suspekte Läsion sonographisch nicht dargestellt werden kann.

In der farbkodierten Duplexsonographie lassen sich tumoröse Gefäßneubildungen durch eine variable Lokalisation und Flussrichtung sowie durch einen nahezu konstanten systolisch-diastolischen Fluss ohne größere Schwankungen charakterisieren [10]. Die Bedeutung der farbkodierten Sonographie bedarf jedoch einer weitergehenden Untersuchung.

Schließlich erlaubt die transthorakale Sonographie, Osteolysen sowie pathologische Frakturen zu identifizieren und im Therapieverlauf zu kontrollieren. Im Rahmen des Tumorstagings gelangen außerdem EUS und EBUS mit Punktion zum Einsatz (siehe oben).

\section{Pneumothorax}

Ein Pneumothorax kann trotz sorgfältiger Untersuchungstechnik als Folge einer transbronchialen Biopsie während der Bronchoskopie und auch nach Pleuraergussdrainage mittels Pleurakatheter entstehen. In Abhängigkeit von der Größe des Pneumothorax ist gegebenenfalls eine unmittelbare Absaugung oder eine Verlaufsbeobachtung erforderlich. Die Inzidenz eines Pneumothorax nach transbronchialer Biopsie wird mit ca. 5,5\% [11] und nach sonographisch gestützter Thorakozentese, in Abhängigkeit von Kathetergröße und Instrumentarium, mit Werten zwischen $2,5 \%$ und $13,9 \%$ angegeben [12-15]. Die sonomorphologischen Befunde beim Vorliegen eines Pneumothorax sind in Tab. 2 aufgelistet. Voraussetzung für eine verlässliche Pneumothoraxdiagnostik ist eine subtile, den gesamten betroffenen Hemithorax erfassende Untersuchungstechnik. Im Unterschied zum einfachen „Pneumothorax“ findet sich beim Seropneumothorax sonographisch entweder ein Luft-Flüssigkeitsspiegel oder ein Pleuraerguss mit multiplen echoreichen Reflexen (im Sinne von Lufteinschlüssen).

Da sich die meisten postinterventionellen Pneumothoraces innerhalb weniger Stunden entwickeln, wird bisher in der klinischen Routine nach 3 Stunden eine Röntgen-Thoraxaufnahme in 
Tab. 2 Sonomorphologische Befunde beim Vorliegen eines Pneumothorax (modifiziert nach [3])

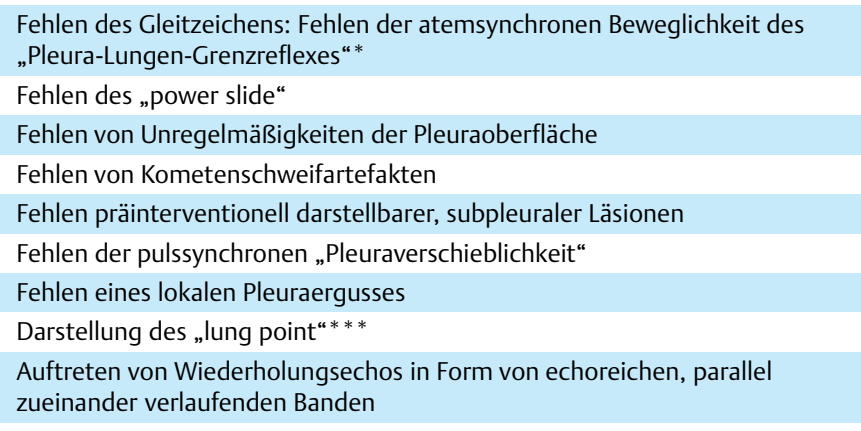

* Der „Pleura-Lungen-Grenzreflex“ wird vereinfacht auch als „Pleura“, „Pleurareflex“ oder „Pleuralinie“ bezeichnet.

** "Power slide“ ist die Darstellung der atemsynchronen Beweglichkeit des „Pleura-Lungen-Grenzreflexes“ mittels Power mode [16].

*** Unter dem „lung point“ wird die Grenze zwischen normal belüfteter Lunge und Pneumothoraxareal verstanden [17].

Exspiration angefertigt. Während große Pneumothoraces in der Regel radiologisch zu identifizieren sind, können kleinere auf der Röntgenaufnahme übersehen werden. Als sensitivste Technik zum Nachweis eines Pneumothorax gilt derzeit die thorakale Computertomographie. Diese Methode bleibt allerdings aufgrund der Strahlenbelastung und der fehlenden therapeutischen Konsequenz bei kleinen pleuralen Luftansammlungen Einzelfällen vorbehalten.

In zwei prospektiven Studien $[18,19]$ wurde der Stellenwert der transthorakalen Sonographie bei der Diagnostik bzw. beim Ausschluss eines Pneumothorax/Seropneumothorax nach transbronchialer Biopsie bzw. Pleuraergussdrainage mittels Pleurakatheter an 1023 bzw. 53 Patienten untersucht. Die Sensitivität der transthorakalen Lungen- und Pleurasonographie betrug dabei 83 bzw. 100\%, die Spezifität jeweils 100\%. Die Treffsicherheit lag bei 99 bzw. 100\%. Diese Ergebnisse belegen, dass auch bei der Diagnostik des postinterventionellen Pneumothorax und Seropneumothorax die transthorakale Sonographie als erste postinterventionelle Methode eingesetzt werden kann (Abb.2) und damit eine Röntgenaufnahme nur noch in bestimmten Situationen erforderlich ist. Hierzu gehören der sonographisch diagnostizierte Pneumothorax zur Bestimmung der Tiefenausdehnung, eine Diskrepanz zum klinischen Befund sowie eine eingeschränkte sonographische Beurteilbarkeit (z. B. bei Vorliegen eines Hautemphysems). Abgesehen von der verminderten Strahlenbelastung, bietet dieses Vorgehen auch die Möglichkeit einer Kostenreduktion.

\section{Diffuse parenchymatöse Lungenerkrankungen}

Bisher wurde der transthorakalen Sonographie keinerlei Bedeutung im Rahmen der Diagnostik diffuser parenchymatöser Lungenerkrankungen (DPLD) beigemessen. Jedoch lassen sich bei DPLD „multiple“ und über die gesamte Lunge verteilte Kometenschweifartefakte in Kombination mit einer verdickten, irregulär/ fragmentierten „Pleuralinie“ nachweisen [20] (Abb.3). Mittels transthorakaler Sonographie gelingt es außerdem, die Verteilung der pleuralen Veränderungen zu erfassen. Des weiteren könnten durch sonographische Verlaufskontrollen mit Quantifizierung

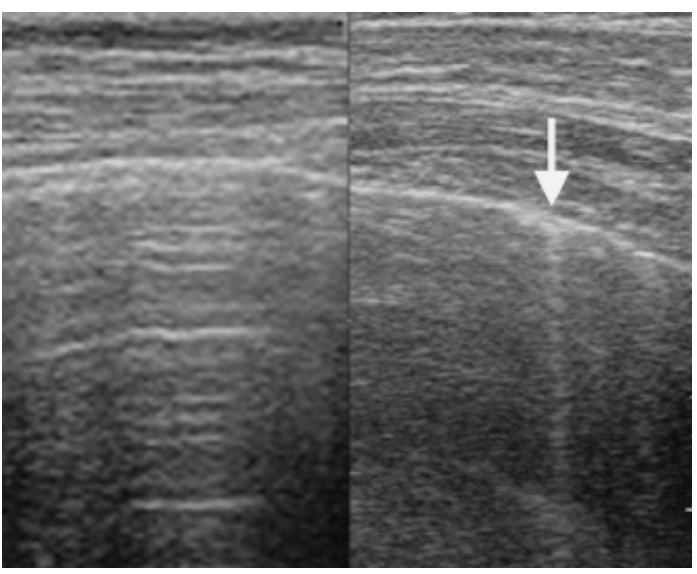

Abb. 2 Rechtsseitiger Pneumothorax - Sonogramm zueinander korrespondierender Interkostalschnitte von ventral (Linearscanner). Rechtsseitiger Pneumothorax bei einer 53-jährigen Patientin mit Pneumonie. Auf der rechten Bildhälfte ist der normale Pleurareflex mit einem Kometenschweifartefakt (Pfeil) als Ausdruck einer vollständig ausgedehnten Lunge dargestellt. Auf der linken Bildhälfte fehlen sowohl der normale Pleurareflex als auch Kometenschweifartefakte. Statt dessen finden sich regelmäßige Wiederholungsechos, die zur Diagnose „Pneumothorax“ führen.

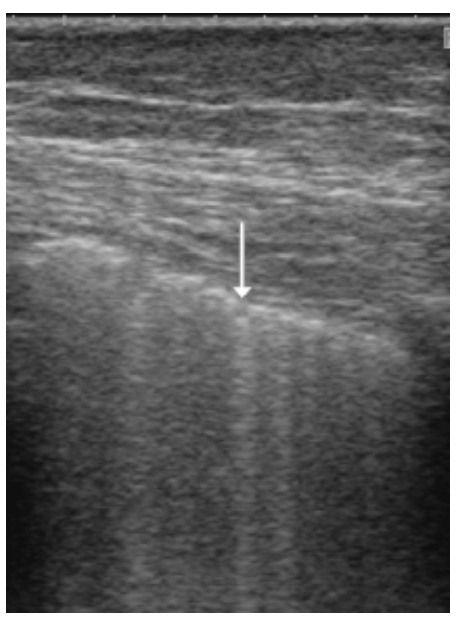

Abb. 3 Lungenfibrose Sonogramm (Linearscanner). 66-jährige Frau mit idiopathischer Lungenfibrose. Die Untersuchung mit dem Linearscanner zeigt eine verdickte, irreguläre/fragmentierte Pleuraoberfläche (Pfeil) mit zahlreichen Kometenschweifartefakten.

der Kometenschweifartefakte je Scan auch Aussagen zur Progredienz bzw. Regredienz von interstitiellen Veränderungen (z.B. unter Therapie) gewonnen werden. Hierbei stellt sich die Frage, ob es sich bei den so genannten Kometenschweifartefakten im Rahmen der DLPD überhaupt um „Artefakte“ im engeren Sinne handelt oder ob hierbei morphologische Veränderungen an der Lungen- und Pleuraoberfläche zur Darstellung kommen. Obwohl auch mittels transthorakaler Sonographie der Verdacht auf Vorliegen einer interstitiellen Lungenerkrankung erhoben und die Verteilung der pleuralen Veränderungen erfasst werden kann, ersetzt die TS bei dieser Fragestellung jedoch keinesfalls die hochauflösende Computertomographie und die histologische Sicherung der Diagnose. Ebenso erlaubt die Sonographie keine Rückschlüsse auf die Genese und Histologie der interstitiellen Veränderungen. 

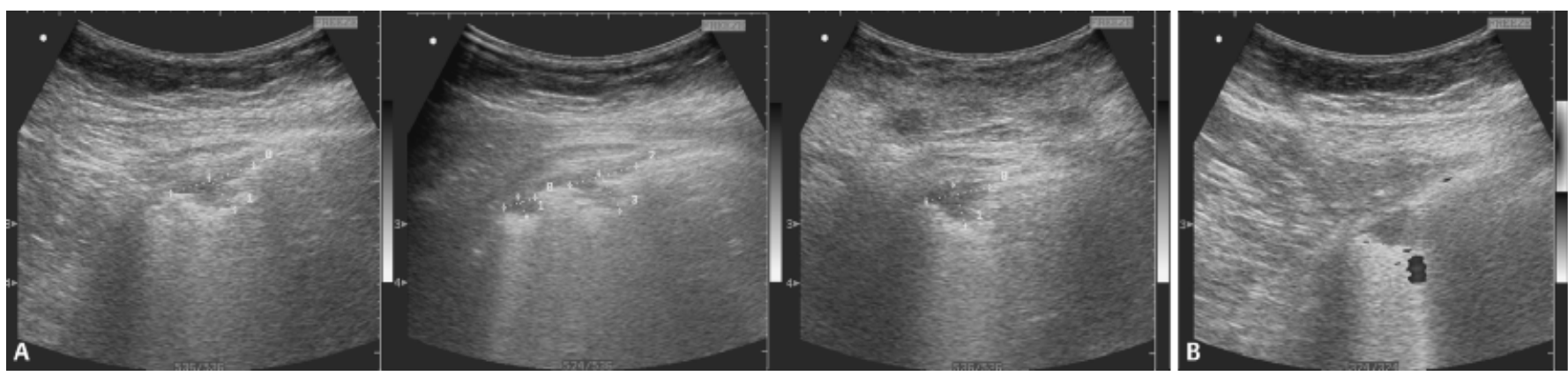

Abb. 4 Lungenembolie - Sonogramm (Konvexscanner). 46-jährige Patientin mit plötzlich aufgetretener progredienter Dyspnoe, verbunden mit rechts-thorakalen atemabhängigen Schmerzen mit Ausstrahlung in den Arm. Mittels transthorakaler Lungen- und Pleurasonographie lassen sich insgesamt vier dreieckförmige echoarme und glatt begrenzte Areale (A) identifizieren, die alle im von der Patientin angegebenen Schmerzbereich lagen. Einer der Herde (B) wies ein „vascular sign“ auf. Mittels „Power-mode“ gelang dabei die Darstellung des Abbruchs des hiluswärts gerichteten, aufgeweiteten und embolisch verschlossenen Pulmonalarteriengefäßes.

\section{Lungenembolie}

Ca. 70 - $80 \%$ der Lungenembolien gehen mit peripheren Veränderungen im Sinne von inkompletten und kompletten Infarkten einher. Charakteristikum der inkompletten Infarkte, die ca. 85\% aller Lungeninfarkte ausmachen, ist, dass sie sich innerhalb von 2-4 Tagen [21 - 22] aufgrund des lytischen Potenzials der Pulmonalgefäße oder auch durch medikamentöse Lyse bzw. Antikoagulation wieder zurückbilden können. Die Lungeninfarkte können sonographisch und auch computertomographisch erfasst werden, wobei aufgrund der o.g. Dynamik thromboembolischer Ereignisse eine zeitgleiche Untersuchung mittels verschiedener Techniken für die Vergleichbarkeit der Ergebnisse gefordert werden muss.

Bisher liegen die Ergebnisse mehrerer lokaler Lungenemboliestudien [23-25] sowie einer multizentrischen Studie [26] zur sonographischen Diagnostik der Lungenembolie vor, die an insgesamt 7 Kliniken in Deutschland und Österreich durchgeführt wurde. Die Sensitivität bzw. Spezifität der transthorakalen Sonographie bei der Diagnostik der Lungenembolie betrug in der multizentrischen Studie unter Berücksichtigung von entweder 2 typischen Läsionen oder einer Läsion mit korrespondierendem Pleuraerguss $74 \%$ bzw. 95\%, die Treffsicherheit lag bei $84 \%$. Der sonographische Befund von zwei oder mehr dreieckförmigen bzw. runden subpleuralen Läsionen (Abb. 4) mit einer pleuralen Basis zwischen 0,5 und $3 \mathrm{~cm}$ kann als Bestätigung einer klinisch vermuteten Lungenembolie angesehen werden (Tab. 3; Abb.5). Der Nachweis einer typischen subpleuralen Läsion in Verbin-

Tab. 3 Sonographische Kriterien der peripheren Veränderungen bei Lungenembolie (modifiziert nach [3])

ein bis mehrere (durchschnittlich 2,3) echoarme, subpleurale Areale
homogene (inhomogene) Echotextur
scharf begrenzt
dreieckförmig oder rund
positive Atemverschieblichkeit
z. T. zentraler Bronchusreflex
lokaler/basaler Pleuraerguss
„vascular sign“*

* Darstellung des Abbruchs des hiluswärts gerichteten, aufgeweiteten und embolisch verschlossenen Pulmonalarteriengefäßes. dung mit einem korrespondierenden Pleuraerguss macht das Vorliegen einer Lungenembolie sehr wahrscheinlich. Für das Vorliegen dieses Kriteriums betrug die Sensitivität $43 \%$ bei einer Spezifität von 99\%. Subpleurale Läsionen mit einer Größe unter 5 Millimetern gelten als unspezifische Veränderungen. Durchschnittlich finden sich sonographisch 2,3 periphere Herde pro Patient mit einer mittleren Größe von 15,5 × 12,4 mm (Spanne 5-62 $\times 5-70 \mathrm{~mm}$ ). Die Hälfte der Patienten weist einen Pleuraerguss auf, in $33 \%$ ist das ein basaler und in $16 \%$ ein lokaler $\mathrm{Er}-$ guss. Die Mehrzahl der peripheren thromboembolischen Läsionen $(66 \%)$ ist in den posterioren basalen Lungensegmenten lokalisiert, die der transthorakalen Sonographie uneingeschränkt zugänglich sind. Damit befinden sich die Ergebnisse der multizentrischen Studie in sehr guter Übereinstimmung mit früheren Studien, die eine Sensitivität und Spezifität zwischen 80-94\% bzw. 87-92\% und eine mittlere Größe der Läsionen zwischen $1,5 \times 2,8 \mathrm{~cm}$ und $1,4 \times 1,1 \mathrm{~cm}$ bei einer durchschnittlichen Anzahl der Herde von 2,6 pro Patient ermittelten $[23,24]$.

Allerdings schließt ein unauffälliger sonographischer Befund eine Lungenembolie nicht aus, da rein zentral lokalisierte Lungenembolien ohne periphere Veränderungen sonographisch nicht erfasst werden können. In diesen Fällen ist der Einsatz weiterer diagnostischer Verfahren erforderlich.

\section{Lungenembolie gesichert: Nachweis von mindestens 2 typischen, dreickförmigen oder runden subpleuralen Läsionen mit einer pleuralen Basis von 0,5-3,0 cm}

2. Lungenembolie wahrscheinlich: Nachweis von einer typischen dreieckförmigen oder runden subpleuralen Läsion mit Pleuraerguss

\section{Subpleurale Läsionen mit pleuraler Bassis $<5 \mathrm{~mm}$ sind unspezifisch}

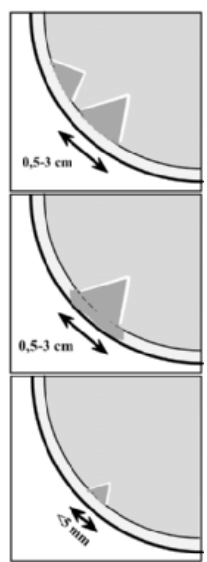

Abb. 5 Diagnostische Kriterien der Lungenembolie. Die Abbildung zeigt die Schlussfolgerungen aus der multizentrischen Studie an 352 Patienten [26]. 


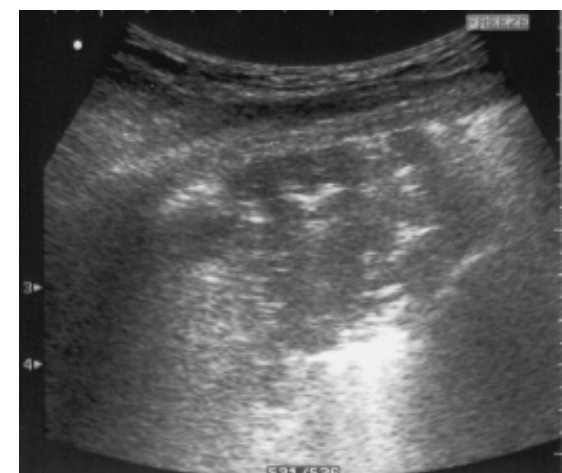

Abb. 6 Pneumonie - Sonogramm (Konvexscanner). 61-jähriger Patient mit Pneumonie. Sonographisch zeigt sich ein unregelmäßig begrenztes echoarmes Areal mit zahlreichen echoreichen Reflexen im Sinne eines positiven Bronchopneumogramms.

\section{Pneumonie}

Eine Pneumonie ist sonographisch nachweisbar, sofern sich das pneumonische Infiltrat in die Lungenperipherie bis hin zur Pleura viszeralis erstreckt. Das für die Pneumonie charakteristische exsudative Infiltrat im betroffenen Lungenareal schafft die Voraussetzung zur Ultraschalldarstellung des betroffenen Lungengewebes. Eine Pneumonie stellt sich sonographisch als echoarmer, unregelmäßig begrenzter Bereich variabler Größe und Form dar. Typisch sind mehrere linsenförmige echoreiche Binnenechos (Abb.6), die von Lufteinschlüssen bzw. luftgefüllten Bronchioli und Bronchien verursacht und als positives Bronchopneumogramm bezeichnet werden. Gelegentlich zeigt sich, als Ausdruck der Sekret-gefüllten Bronchien, ein Fluidobronchogramm, welches sonographisch als echolose, verzweigte tubuläre Struktur ohne Perfusionssignal in Erscheinung tritt. Ein Fluidobronchogramm tritt vor allem bei einer poststenotischen Pneumonie auf, und dieser Verdacht bedarf einer weitergehenden Abklärung. Sehr häufig kommt es im Rahmen der Pneumo- nie zu einer Pleurabeteiligung, die sonographisch als lokalisierte oder basale Ergussbildung darstellbar ist. Tritt im Verlauf der Pneumonie eine Einschmelzung bzw. Abszessbildung auf, so ist diese sonographisch als echolose Zone innerhalb des Infiltrates erfassbar.

Das sonomorphologische Bild der Pneumonie verändert sich im Verlaufe der Erkrankung. Im Stadium der Hepatisation (z. B. bei unbehandelter Lobärpneumonie) verlieren sich die Luftreflexe innerhalb der pneumonischen Läsion. Vielmehr ist dann der sonographische Befund durch ein homogenes, hypodenses, unregelmäßig konturiertes Areal charakterisiert, welches in Echogenität und Echostruktur dem sonographischen Bild der Leber ähnelt. Mit zunehmender Wiederbelüftung nehmen die echoreichen Lufteinschlüsse und Kometenschweifartefakte kontinuierlich zu. Farbdopplersonographisch weisen pneumonische Infiltrate ein verstärktes reguläres Perfusionsmuster auf.

Die Primärdiagnostik der Pneumonie erfolgt nach wie vor klinisch und radiologisch. Bei typischem klinischen und sonographischen Befund könnte jedoch auch eine erst am Ende der Therapie durchgeführte Röntgenaufnahme zur Dokumentation des Therapieerfolges ausreichen. Daneben sind die Darstellung parapneumonischer Pleuraergüsse und die frühzeitige Erfassung von Komplikationen, wie z.B. nekrotisierende bzw. abszedierende Verlaufsformen, die Domäne der transthorakalen Sonographie. Sie ist ferner die Methode der Wahl bei Verlaufskontrollen von sonographisch erfassbaren Pneumonien und reduziert somit die Zahl radiologischer Kontrollen. In Abb. 7 sind die wichtigsten sonographischen Kriterien zur Differenzierung von Bronchialkarzinomen, Lungenembolien und Pneumonien dargestellt.

\section{Pleuraerguss}

Die transthorakale Sonographie ist die sensitivste Methode zur Darstellung von Pleurergüssen. Mit hochauflösenden Ultraschallgeräten können selbst die physiologisch sich im Pleura-

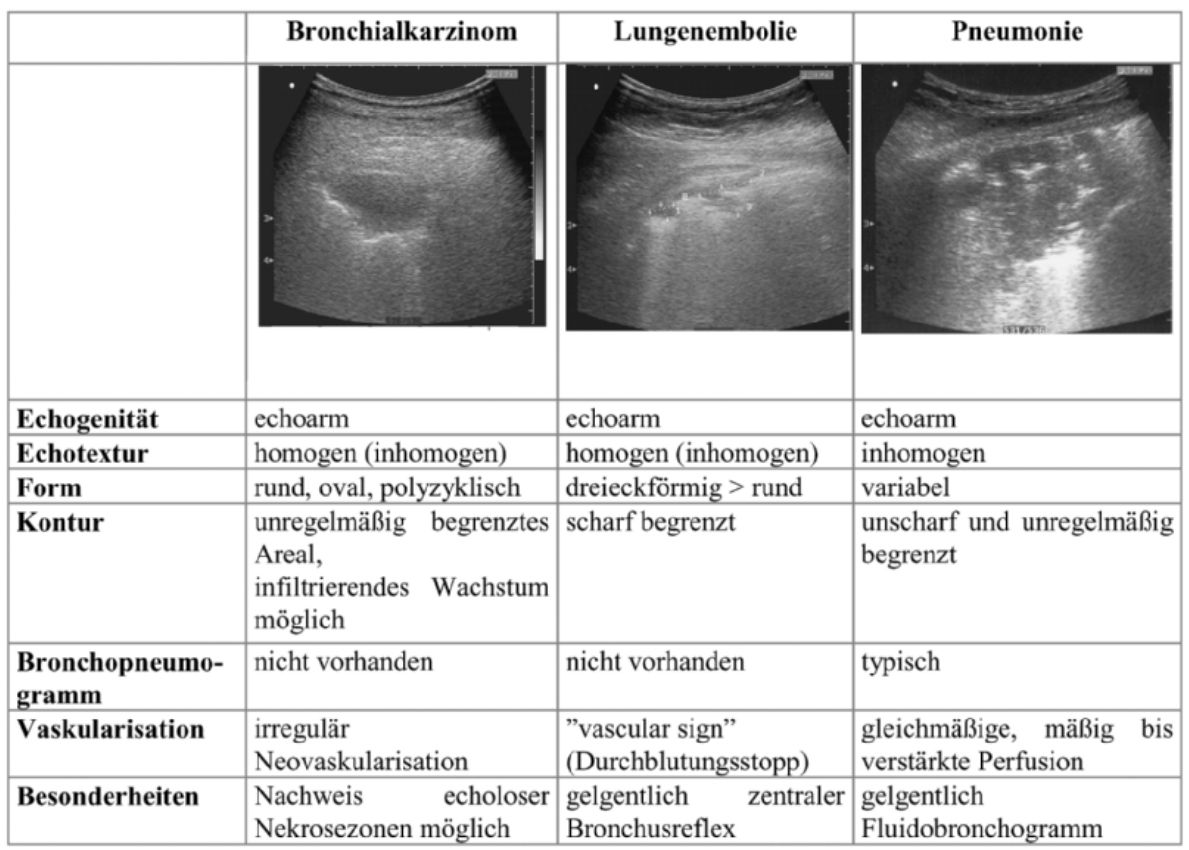

Abb. 7 Diagnoseweisende sonographische Kriterien zur Differenzierung von Bronchialkarzinom, Lungenembolie und Pneumonie (modifiziert [4]). 


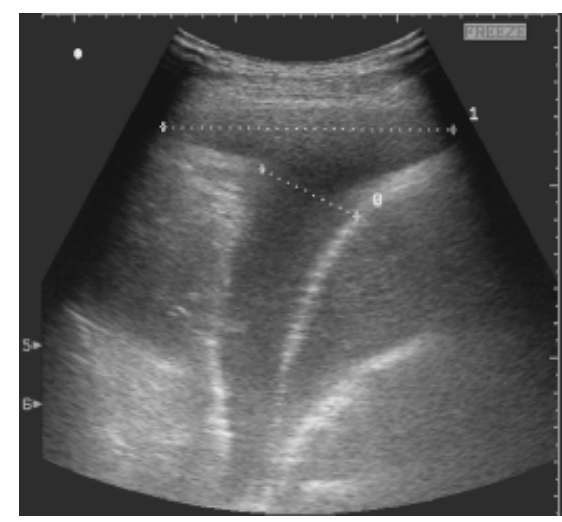

Abb. 8 Schätzung der Pleuraergussmenge Sonogramm (Konvexscanner). Volumetrisch wird die Ergussmenge (in Millilitern) geschätzt, indem beim sitzenden Patienten die maximale Ergusshöhe (1) in $\mathrm{cm}$ und der basale Lungen-Zwerchfellabstand $(0)$ in $\mathrm{cm}$ addiert und anschließend mit der Konstante $\mathrm{k}(\mathrm{k}=70)$ multipliziert werden.
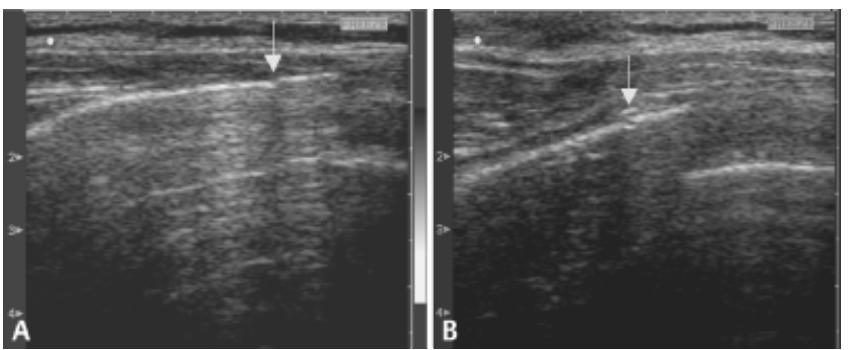

Abb. 9 Rippenfraktur - Sonogramm (Linearscanner). 46-jähriger Patient mit thorakalen Schmerzen rechts ventral nach einem Trauma. Im Schmerzbereich der 5. Rippe rechts zeigt sich eine nicht dislozierte Rippenfraktur mit Unterbrechung des Kortikalisreflexes (Pfeil) und angedeutetem „Kaminphänomen“ (A). Vier Wochen nach dem Ereignis stellt sich sonographisch eine echoreiche Überbrückung des Frakturspaltes (Pfeil) im Sinne einer Kallusbildung dar (B).

spalt befindlichen geringen Flüssigkeitsmengen dargestellt werden. Als Faustregel gilt, dass Transsudate in der Regel echofrei, Exsudate demgegenüber mäßig echogen oder echofrei sind. Da jedoch auch echogene Transsudate vorkommen, ist nur mittels sonographisch gestützter Punktion mit nachfolgender laborchemischer Ergussdiagnostik die Differenzierung zwischen Transsudat und Exsudat sicher möglich. Zur Schätzung des Ergussvolumens (in Millilitern) beim sitzenden Patienten (Abb. 8) wird der basale Lungen-Zwerchfell-Abstand zur maximalen Ergusshöhe (jeweils in $\mathrm{cm}$ ) addiert und anschließend mit 70 multipliziert [3].

\section{Läsionen des knöchernen Thorax}

Neuere Untersuchungen zeigen, dass im Vergleich zur RöntgenThoraxaufnahme sonographisch bis zu 10-mal mehr Rippenfrakturen diagnostiziert werden können [27]. Der wesentliche Vorteil der Sonographie besteht vor allem darin, dass entsprechend der vom Patienten angegebenen Schmerzlokalisation eine dynamische Untersuchung möglich ist. So lassen sich die Veränderungen, die sich bei leichtem Druck auf den Frakturbereich bzw. die schmerzhafte Stelle ergeben, beobachten. Dabei kann auch das Ausmaß der Rippendislokation bestimmt werden (Abb.9). Die typischen sonographischen Befunde bei Knochenfrakturen und Knochenmetastasen sind in den Tab. 4 und $\mathbf{5}$ zusammengefasst.

\section{Farbdopplersonographie bei pulmonalen Läsionen}

Innerhalb pulmonaler Läsionen können mit modernen Ultraschallgeräten bei sehr empfindlicher Einstellung und subtiler Untersuchungstechnik neben der Unterscheidung zwischen ar-

Tab. 4 Direkte und indirekte sonographische Befunde bei Knochenfrakturen (modifiziert nach [3])

Direkte sonographische Befunde

Dehiszenz oder Stufenbildung im Kortikalisreflex

- Dehiszenz oder Stufenbildung im mit dem Schallkopf
Kortikalisreflex bei leichtem Druck

Indirekte sonographische Befunde

- „Kaminphänomen“*

- Weichteilschwellung

- lokales Hämatom

- korrespondierender Pleuraerguss

- gegebenenfalls posttraumatischer Pneumothorax

- gegebenenfalls posttraumatische Lungenkontusionsherde

* Als „Kaminphänomen“ wird ein Artefakt bezeichnet, der sich im Frakturspalt senkrecht in die Tiefe projiziert.

Tab. 5 Sonographische Befunde bei Knochenmetastasen (modifiziert nach [3])

Kortikalisunregelmäßigkeiten

Kortikalisverschmälerung

Kortikalisunterbrechung

pathologische Schalltransmission

echoarme Strukturen mit irregulär eingestreuten Reflexen im Markraum Infiltration periossärer Weichteile

Periostanhebung

\begin{tabular}{|c|c|c|c|c|}
\hline & Pulmonalarterien & Interkostalarterien & Bronchialarterien & $\begin{array}{l}\text { Tumoröse Gefäß- } \\
\text { neubildungen }\end{array}$ \\
\hline $\begin{array}{l}\text { Flußcha- } \\
\text { rakteristik }\end{array}$ & $\begin{array}{l}\text { hoher } \\
\text { Flußwiderstand, } \\
\text { überwiegend } \\
\text { triphasisch }\end{array}$ & $\begin{array}{l}\text { hoher } \\
\text { Flußwiderstand, } \\
\text { monphasisch }\end{array}$ & $\begin{array}{l}\text { niedriger } \\
\text { Flußwiderstand, } \\
\text { monphasisch }\end{array}$ & $\begin{array}{l}\text { fast konstanter } \\
\text { Fluß ohne } \\
\text { systolisch- } \\
\text { diastolische } \\
\text { Variation }\end{array}$ \\
\hline Flußrichtung & zentrifugal & horizontal & variabel & variabel \\
\hline Lokalisation & variabel & horizontal & variabel & variabel \\
\hline Besonderheiten & $\begin{array}{l}\text { hypoxische } \\
\text { Vasokonstriktion }\end{array}$ & & & $\begin{array}{l}\text { langsame Fluß- } \\
\text { geschwindigkeit }\end{array}$ \\
\hline $\begin{array}{l}\text { Schematische } \\
\text { Darstellung }\end{array}$ & & & & \\
\hline
\end{tabular}

Abb. 10 Dopplersonographische Charakterisierung der Durchblutung von pulmonalen Läsionen (modifiziert nach [10]).

teriellen und venösen Flusssignalen folgende Gefäße differenziert werden: 1) Interkostalarterien, 2) Bronchialarterien, 3) Pulmonalarterien und 4) tumoröse Gefäßneubildungen [10] (Abb. 10). Im Bereich von lokalisierten Pleuraergüssen, Lungenzysten und Lungenemboliearealen ist keine Durchblutung nachweisbar. Auch sehr niedrige Flussgeschwindigkeiten (unterhalb von $2 \mathrm{~cm} /$ Sekunde) entziehen sind der Darstellung, da sie unterhalb der Sensitivität des Ultraschallempfängers liegen. Eine reduzierte Durchblutung mit einer zerstörten Gefäßarchitektur ist für Karzinome und maligne Lymphome charakteristisch. Dagegen weisen benigne Läsionen, wie z.B. Pneumonien und Atelektasen, eine verstärkte, baumartige Vaskularisation auf [10]. Die Aussagefähigkeit der Farbdopplersonographie kann möglicher- 
weise durch den Einsatz moderner Ultraschallkontrastmittel noch verbessert werden.

Fasst man die oben skizzierten Möglichkeiten der Thoraxsonographie zusammen, ist es nicht schwer vorauszusagen, dass die vielfältigen Einsatzmöglichkeiten der Sonographie im Bereich des Thorax in Zukunft das diagnostische und therapeutische Vorgehen in der Pneumologie wesentlich erweitern werden.

\section{Literatur}

${ }^{1}$ Reißig A, Kroegel C. Therapeutisches Vorgehen bei akuter Lungenembolie. Internist 2004; 45: 540-548

2 Konstantinides S, Hasenfuß G. Akutes Cor pulmonale bei Lungenembolie. Entscheidender prognostischer Faktor und kritischer Parameter für die Auswahl der therapeutischen Strategie. Internist 2004; 45: $1155-1162$

${ }^{3}$ Kroegel C, Reißig A. Transthorakale Sonographie. Grundlagen und Anwendungen. Einführung und Leitfaden für die Praxis mit CD-ROM. 1. Auflage. Stuttgart, New York: Georg Thieme, 2000

${ }^{4}$ Reißig A, Kroegel C. Ultrasound of the lung and pleura. Chapter 7. In: Gibson GJ, Geddes DM, Costabel U, Sterk PJ, Corrin B (eds.). Respiratory Medicine, third edition. London, New York: Harcourt Health Science, 2003: $370-377$

${ }^{5}$ Mathis G. Thoraxsonographie - Teil 1: Brustkorb und Pleura. Praxis 2004; 93: 615-621

${ }^{6}$ Mathis G. Thoraxsonographie - Teil 2: Subpleurale Läsionen. Praxis 2004; 93: $791-824$

${ }^{7}$ Nakano N, Yasumitsu T, Kotake Y et al. Preoperative histologic diagnosis of chest wall invasion by lung cancer using ultrasonically guided biopsy. J Thor Cardiovasc Surg 1994; 107: 891 - 895

${ }^{8}$ Beckh S, Bölcskei PL. Biopsie thorakaler Raumforderungen - Von der computertomographischen zur ultraschallgezielten Punktion. Ultraschall in Med 1997; 18: 220-225

${ }^{9}$ Mathis G, Bitschnau R, Gehmacher $O$ et al. Ultraschallgeführte transthorakale Punktion. Ultraschall in Med 1999; 20: 226-235

${ }^{10}$ Görg C, Bert T. Transcutaneous color Doppler sonography of lung consolidations. Review and pictorial essay. Part 1: Pathophysiologic and colour Doppler sonographic basics of pulmonary vascularity. Ultraschall in Med 2004; 25: $221-226$

${ }^{11}$ Herf SM, Suratt PM, Arosa NS. Deaths and complications associated with transbronchial lung biopsy. Am Rev Respir Dis 1977; 115: $708-711$
${ }^{12}$ Raptopoulos V, Davis LM, Lee G et al. Factors affecting the development of pneumothorax associated with thoracentesis. AJR 1991; 156: 917-920

${ }^{13}$ Aleman C, Alegre J, Armadans L et al. The value of chest roentgenology in the diagnosis of pneumothorax after thoracentesis. Am J Med 1999; 107: $340-343$

${ }^{14}$ Peterson WG, Zimmermann R. Limited utility of chest radiograph after thoracentesis. Chest 2000; 117: 1038 - 1042

15 Jones PW, Moyers JP, Rogers JT et al. Ultrasound-guided thoracentesis. Is it a safer method? Chest 2003; 123: $418-423$

${ }^{16}$ Cunningham J, Kirkpatrick AW, Nicolaou $S$ et al. Enhanced recognition of "lung sliding" with power color Doppler imaging in the diagnosis of pneumothorax. J Trauma 2002; 52: 769-771

${ }^{17}$ Lichtenstein D, Meziere G, Biderman P et al. The "lung point": an ultrasound sign specific to pneumothorax. Intensive Care Med 2000; 26: $1434-1440$

${ }^{18}$ Herth FJF, Eberhardt R, Ernst A et al. Diagnosis of pneumothorax (PTX) by means of transthoracic ultrasound - a prospective trial. Eur Respir J 2004; 24 (Suppl. 48): S491

19 Reißig A, Kroegel C. Accuracy of transthoracic sonography in excluding post-interventional pneumothorax and hydropneumothorax. Comparison to chest radiography. Eur J Radiol 2005; 53: 463-470

${ }^{20}$ Reißig A, Kroegel C. Transthoracic sonography of diffuse parenchymal lung disease. The role of comet tail artifacts. J Ultrasound Med 2003; 22: $173-180$

${ }^{21}$ Hampton AO, Castleman B. Correlation of postmortem chest teleroentgenograms with autopsy findings. Am J Roentgenol Radium Ther 1940; 43: $305-326$

${ }^{22}$ Kroegel C, Reißig A. Principle mechanisms underlying venous thromboembolism: epidemiology, risk factors, pathophysiology and pathogenesis. Respiration 2003; 70: 7-30

${ }^{23}$ Mathis G, Bitschnau R, Gehmacher O et al. Chest ultrasound in diagnosis of pulmonary embolism in comparison to helical CT. Ultraschall in Med 1999; 20: 54-59

${ }^{24}$ Reißig A, Heyne J-P, Kroegel C. Sonography of lung and pleura in pulmonary embolism: sonomorphologic characterization and comparison with spiral CT scanning. Chest 2001; 120: 1977-1983

${ }^{25}$ Lechleitner P, Riedl B, Raneburger W et al. Chest sonography in the diagnosis of pulmonary embolism: a comparison with MRI angiography and ventilation perfusion scintigraphy. Ultraschall in Med 2002; 23: $373-378$

${ }^{26}$ Mathis G, Blank W, Reißig A et al. Thoracic ultrasound for diagnosing pulmonary embolism - a prospective multicenter study of 352 patients (TUSPE). Chest, 2005 in press

${ }^{27}$ Griffith JF, Rainer TH, Ching ASC et al. Sonography compared with radiography in revealing acute rib fracture. AJR 1999; 173: 1603 -1609 